\title{
AN EMBEDDING THEOREM FOR CERTAIN SPACES WITH AN EQUIDISTANT PROPERTY
}

SAM B. NADLER, JR. ${ }^{1}$

\begin{abstract}
It is shown that certain metric spaces with the unique equidistant property can be topologically embedded in the real line. Several examples are given which show that the spaces considered are nontrivial, and which indicate that the technique of proof is necessary.
\end{abstract}

1. Introduction. A metric space $(X, d)$ is said to have the unique equidistant property, written $(X, d) \in \mathrm{UEP}$, provided that if $a, b \in X$ with $a \neq b$, then there exists one and only one point $x \in X$ such that $d(a, x)=d(b, x)$; the point $x$ will be denoted by $E(a, b)$ and the function $E:[X \times X] \backslash \Delta \rightarrow X$ will be called the equidistant function [ $\Delta$ denotes the diagonal of $X \times X]$.

In [1], where UEP was denoted UMP, Berard showed that a connected metric space with UEP is topologically a connected subset of the reals $R^{1}$. The main purpose of this note is to prove that any locally compact separable metric space having UEP is topologically embeddable in $R^{1}$ (see (2.5)). A second purpose is to give examples of such spaces which are neither connected nor totally-disconnected. Also, an example is given of a metric space having UEP which is not embeddable in $R^{1}$.

Throughout this paper the symbol $\times$ denotes cartesian product and the slash " " denotes complementation for sets. The word nondegenerate means consisting of more than one point.

For other papers related to material here and in [1] see, for example, [2] and [5] through [9]. In particular, I first became interested in these subjects when I read the lovely article by Loveland and Wayment [9].

2. Results. The main result is (2.5). First, some lemmas.

(2.1) LemMA. If $C$ is a nondegenerate connected subset of $(X, d) \in \mathrm{UEP}$, then $E([C \times C] \backslash \Delta) \subset C$.

Proof. Assume $a, b \in C$ such that $a \neq b$. Define $f: C \rightarrow R^{1}$ by $f(y)$

Received by the editors December 7, 1975.

AMS (MOS) subject classifications (1970). Primary 54E99; Secondary 54F99.

Key words and phrases. Dimension, locally compact, one-point compactification, separability, totally-disconnected.

${ }^{1}$ This paper was written while the author was a visitor at the University of Delaware, Newark, Delaware. 
$=d(a, y)-d(b, y)$ for each $y \in C$. Clearly $f$ is continuous and $f(a)<0$ while $f(b)>0$. Hence, since $C$ is connected, there exists $y_{0} \in C$ such that $f\left(y_{0}\right)$ $=0$, i.e., $d\left(a, y_{0}\right)=d\left(b, y_{0}\right)$. Therefore, $E(a, b)=y_{0} \in C$.

(2.2) Remark. Lemma 2.1 implies UEP is inherited by connected subsets.

(2.3) Lemma. Let $Y=A \cup\left\{p_{1}, p_{2}, \ldots, p_{n}, \ldots\right\}$ be a compactum consisting of an arc $A$ with endpoints $e_{1}$ and $e_{2}$ and a sequence of points $p_{n} \notin A$ such that $p_{n} \rightarrow p \in\left[A \backslash\left\{e_{1}, e_{2}\right\}\right]$. Then $Y$ cannot be a subspace of any $(X, d) \in \mathrm{UEP}$.

Proof. Suppose $Y \subset X$ and $(X, d) \in \mathrm{UEP}$. Choose $k$ large enough so that $d\left(p_{k}, e_{i}\right)>d\left(p_{k}, p\right)$ for each $i=1$ and 2. Choose $\varepsilon>0$ such that, for each $i=1$ and 2 ,

$$
d\left(p_{k}, p\right)<\varepsilon<d\left(p_{k}, e_{i}\right) .
$$

Now, for each $i=1$ and 2, let $A_{i}$ denote the subarc of $A$ with endpoints $e_{i}$ and $p$. By $(*)$ and the connectedness of each $A_{i}$, there exists $a_{i} \in A_{i}$ such that $d\left(p_{k}, a_{i}\right)=\varepsilon$ for each $i=1$ and 2. Also $a_{1} \neq a_{2}$, otherwise $a_{1}=a_{2} \in\left[A_{1}\right.$ $\left.\cap A_{2}\right]=\{p\}$ and therefore $d\left(p_{k}, a_{i}\right)=d\left(p_{k}, p\right)<\varepsilon$. Hence, $E\left(a_{1}, a_{2}\right)=p_{k}$ $\notin A$ which is a contradiction to (2.1).

The next lemma is a direct consequence of [1, Corollary 13] and (2.2) above.

(2.4) Lemma. If $(X, d) \in \mathrm{UEP}$, then any component of $X$ must be homeomorphic to a connected subset of $R^{1}$.

(2.5) THEOREM. If $(X, d)$ is a locally compact separable metric space such that $(X, d) \in \mathrm{UEP}$, then $X$ is topologically embeddable in $R^{1}$.

Proof. The proof is the same as the proof of Theorem 2 in [4, pp. 9-10], except:

(i) Lemma 2.3 above replaces Lemma 2.2 of [4];

(ii) Lemma 2.4 above replaces Lemma 5.1 of [4].

One comment about the proof of Theorem 2 in [4] that may be helpful: On p. 10 of [4] we showed that a certain one-point compactification $N^{*}$ satisfies (1) and (2) of Theorem 1 of [10]. We did not show that $N^{*}$ is embeddable in some Euclidean $n$-space, which is one of the hypotheses in Theorem 1 of [10]. However, if a compact metric space satisfies (1) and (2) of Theorem 1 of [10], then it is embeddable in Euclidean 3-space. This follows from the fact that any compact metric space $X$, each component of which is of dimension $\leqslant 1$, is itself of dimension $\leqslant 1$ (let $\nu: X \rightarrow Y$ denote the natural map of $X$ onto the compact 0-dimensional decomposition space of $X$ into the components of $X$, and apply [3, p. 92] to $\nu$ ).

3. Examples. Throughout this section let $R^{2}$ denote the Euclidean plane with the usual metric $\rho$,

$$
\rho\left(\left(x_{1}, y_{1}\right),\left(x_{2}, y_{2}\right)\right)=\left[\left(x_{1}-x_{2}\right)^{2}+\left(y_{1}-y_{2}\right)^{2}\right]^{1 / 2}
$$


for each $\left(x_{1}, y_{1}\right),\left(x_{2}, y_{2}\right) \in R^{2}$. I will also use the symbol $\rho$ to denote the metric inherited by any subset of $R^{2}$.

For each of the examples of metric spaces having UEP, given so far in the literature, the underlying space has been either connected or totally-disconnected. I will give some examples of metric spaces with UEP which are neither connected nor totally-disconnected. As the reader will see, the most important examples for our purposes will be (3.2) and (3.3). However, the following simple example is included because some of its features illustrate the necessity for (3.2) and (3.3).

(3.1) EXAMPLE. Let

$$
X=\left\{\left(x, x^{2}\right) \in R^{2}: x \geqslant 0\right\} \cup\{(-1,+1)\} .
$$

Then, $(X, \rho)$ is a locally compact separable complete metric space having UEP which is neither connected nor totally-disconnected.

Now, let $(X, d)$ be any locally compact separable metric space having UEP such that $X$ also has the following two properties (which the space of (3.1) has):

(P.1) each nondegenerate component of $X$ is an open subset of $X$;

(P.2) the union of the one-point components of $X$ is an open subset of $X$.

Then a much simpler argument than that given for (2.5) can be given to show $X$ is embeddable in $R^{1}$. We sketch it briefly. By separability, (2.3), and (2.4) there are only countably many nondegenerate components of $X$. Thus, using (2.4) to embed different nondegenerate components in mutually disjoint subintervals of $(-\infty,-1)$, we obtain by (P.1) an embedding $e_{1}$ of the union of the nondegenerate components of $X$ into $(-\infty,-1)$. By (P.2) and [3, Remark 1 , p. 22], the rest of $X$ is (at most) 0 -dimensional and therefore, by [3, p. 60], may be embedded in $[0,1]$ by an embedding $e_{2}$. Then, (P.1) and (P.2) allow us to paste $e_{1}$ and $e_{2}$ together to obtain an embedding of all of $X$ into $R^{1}$.

Thus, we need examples of locally compact separable metric spaces having UEP, but not satisfying (P.1) and (P.2), in order to indicate that the use of Theorem 1 of [10], in the proof of (2.5), was necessary. I now give two such examples.

(3.2) ExAmple. We use polar coordinates. For each $n=1,2, \ldots$, let

$$
\begin{aligned}
A_{n} & =\left\{(1, \theta):-\pi \cdot 2^{1-2 n} \leqslant \theta \leqslant-\pi \cdot 2^{-2 n}\right\}, \\
B_{n} & =\left\{(1, \theta): \pi-\pi \cdot 2^{-2 n}<\theta<\pi-\pi \cdot 2^{-2 n-1}\right\}, \\
C & =\{(1, \theta): 0 \leqslant \theta<\pi / 2\} .
\end{aligned}
$$

Now, let

$$
X=\left[\bigcup_{n=1}^{\infty} A_{n}\right] \cup\left[\bigcup_{n=1}^{\infty} B_{n}\right] \cup C .
$$

The fact that $(X, \rho) \in$ UEP can easily be seen from the following simple 
observation. If $\left(1, \theta_{1}\right)$ and $\left(1, \theta_{2}\right)$ are any two points on the unit circle, then the two points equidistant from them on the circle are diametrically opposite to one another; in fact, they are $\left(1,\left(\theta_{1}+\theta_{2}\right) / 2\right)$ and $\left(1,\left(\theta_{1}+\theta_{2}\right) / 2+\pi\right)$. It follows that $(X, \rho)$ is a locally compact separable metric space having UEP but not satisfying (P.1) (the component $C$ is not an open subset of $X$ ).

(3.3) Example. For each $n=1,2, \ldots$, let $A_{n}$ and $B_{n}$ be as in (3.2). Using polar coordinates, let

$$
D=\{(1, \theta): \pi<\theta<3 \pi / 2\}
$$

Now let

$$
X=\left[\bigcup_{n=1}^{\infty} A_{n}\right] \cup\left[\bigcup_{n=1}^{\infty} B_{n}\right] \cup D \cup\{(1,0)\} .
$$

It is easy, using the observation about the unit circle in (3.2), to see that $(X, \rho) \in \mathrm{UEP}$. It follows that $(X, \rho)$ is a locally compact separable metric space having UEP but not satisfying (P.2) (the only one-point component of $X$ is $\{(1,0)\}$, and it is not an open subset of $X)$.

Thus, (3.2) and (3.3) show the necessity for the use of Theorem 1 of [10] in the proof of (2.5). They also show that the spaces considered in the hypothesis of (2.5) are interesting and nontrivial in that they do not have to satisfy (P.1) and (P.2).

My last example shows that not every metric space having UEP is embeddable in $R^{1}$. In fact, it is a locally compact metric space having UEP which is not separable.

(3.4) Example. Let $(X, \sigma)$ be any uncountable metric space having UEP. Change the metric $\sigma$ to the metric $d$ defined by the following formulas:

$$
\begin{aligned}
& d(x, y)=1+\sigma(x, y) \quad \text { for } x, y \in X \text { with } x \neq y \\
& d(x, y)=0 \quad \text { if and only if } x=y
\end{aligned}
$$

It is easy to verify that $(X, d)$ is a metric space and that $(X, d)$ is discrete (i.e., $\{x\}$ is an open subset of $X$ for each $x \in X)$. It is also easy to see that, for any $x, y, z \in X, d(x, z)=d(y, z)$ is equivalent to $\sigma(x, z)=\sigma(y, z)$. From this equivalence and the fact that $(X, \sigma) \in \mathrm{UEP}$, it follows easily that $(X, d)$ $\in \operatorname{UEP}$. Since $(X, d)$ is uncountable and discrete, $(X, d)$ is not separable and (hence) is not embeddable in $R^{1}$.

(3.5) Problem. There are many examples of totally-disconnected separable metric spaces having UEP (the rationals with absolute-value giving the metric, a three-point discrete space with equal distance between any two distinct points, Example 2 of [1], etc.). All the totally-disconnected separable metric spaces I know of which have UEP are 0-dimensional. Since there are totallydisconnected separable metric spaces of arbitrary dimension (see the footnote in [3, p. 23]), the following question arises: Must a totally-disconnected separable metric space having UEP be 0-dimensional? If the answer is "yes", then such spaces are embeddable in $R^{1}$ [3, p. 60]. 


\section{REFERENCES}

1. Anthony D. Berard, Jr., Characterizations of metric spaces by the use of their midsets: Intervals, Fund. Math. 73 (1971/72), 1-7. MR 45 \#4368.

2. A. D. Berard, Jr. and W. Nitka, A new definition of the circle by the use of bisectors, Fund. Math. 85 (1974), 49-55. MR 50 \#8464.

3. W. Hurewicz and H. Wallman, Dimension theory, Princeton Univ. Press, Princeton, N.J., 1941. MR 3, 312.

4. K. Kuratowski, Sam B. Nadler, Jr. and G. S. Young, Continuous selections on locally compact separable metric spaces, Bull. Acad. Polon Sci. Ser. Sci. Math. Astronom. Phys. 18 (1970), 5-11. MR 41 \# 9221.

5. A. Lelek and W. Nitka, On convex metric spaces. I, Fund. Math. 49 (1960/61), 183-204. MR 23 \# A2192.

6. L. D. Loveland and J. E. Valentine, Characterizing a circle with the double midset property, Proc. Amer. Math. Soc. 53 (1975), 443-444.

7. - Convex metric spaces with 0-dimensional midsets, Proc. Amer. Math. Soc. 37 (1973), 568-571. MR 46 \#9915.

8. - Generalized midset properties characterize geodesic circles and intervals (preprint).

9. L. D. Loveland and S. G. Wayment, Characterizing a curve with the double midset property, Amer. Math. Monthly 81 (1974), 1003-1006.

10. Edwin W. Miller, On subsets of a continuous curve which lie on an arc of the continuous curve, Amer. J. Math. 54 (1932), 397-416.

Department of Mathematics, University of Delaware, Newark, Delaware 19711

Department of Mathematics, University of Kentucky, Lexington, Kentucky 40506 